\title{
Galactic cosmic rays
}

\author{
Pasquale Blasi ${ }^{1,2, a}$ \\ 1 INAF/Osservatorio Astrofisico di Arcetri, Largo E. Fermi 5, 50125 Firenze, Italy \\ 2 Gran Sasso Science Institute (INFN), Viale F. Crispi 6, 60100 L'Aquila, Italy
}

\begin{abstract}
The multi-facet nature of the origin of cosmic rays is such that some of the problems currently met in our path to describing available data are due to oversimplified models of CR acceleration and transport, and others to lack of knowledge of the physical processes at work in certain conditions. On the other hand, the phenomenology of cosmic rays, as arising from better observations, is getting so rich that it makes sense to try to distinguish the problems that derive from too simple views of Nature and those that are challenging the very foundations of the existing paradigms. Here I will briefly discuss some of these issues.
\end{abstract}

\section{Introduction}

The perceived complexity of a physical problem often depends on how close we observe it. The origin of cosmic rays (CRs) is no exception to this rule: generally speaking it is fair to say that at present there is little doubt in the Physics community at large, that the bulk of CRs are produced in the Milky way, and that supernova (SN) explosions have something to do with it, mainly through the formation of strong shock waves. Even the simplest approach to diffusive shock acceleration (DSA) delivers power law spectra of CRs with slopes that are not too different from the ones that are required based on observations of the multifrequency spectrum of supernova remnants (SNRs). There is also little doubt that the motion of CRs throughout the Galaxy is generally diffusive in nature, and that diffusion occurs in an energy dependent manner so as to reproduce the fact that the nuclear secondary-to-primary ratios decrease with energy above a few tens $\mathrm{GeV} / \mathrm{n}$. At this level, we can say that there is a general understanding of the CR origin in the Galaxy, namely we do have a model for their acceleration (DSA) in SNR shocks and we have an understanding of the transport of CRs through the Galaxy.

If one tries to get a closer look at the problem, it appears rather more complex and interesting. For instance, DSA returns spectra of accelerated particles at strong shocks that are invariably $f(p) \propto p^{-4}$ (namely $\propto E^{-2}$ at relativistic energies), but often gamma ray observations appear to require steeper spectra [1]. Moreover, nonlinear corrections to DSA lead to concave spectra [2], harder than $p^{-4}$ at high energy, namely even more at odds with multifrequency observations [3]. The problem of the maximum energy of accelerated particles adds to the problem: in order to achieve rigidities as high as a few million GV (the knee), substantial magnetic field amplification is necessary, and our current understanding of this mechanism requires sizeable currents of high

a e-mail: blasi@arcetri.astro.it energy escaping particles, which in turn reflect into a need for hard spectra. Magnetic field amplification seems to be better understood in terms of the so-called non-resonant hybrid instability [4], but even when this process is at work the highest energy achievable in a type Ia SNR is only $\sim 200 \mathrm{TeV}$ [5-7], while energies as high as $\sim \mathrm{PeV}$ might be reachable in type II $\mathrm{SN}$ explosions in the dense winds of their red giant parent stars, if the spectrum of accelerated particles is relatively hard ( $\propto p^{-4}$ or flatter). On the other hand, one could argue that steep spectra might be better reconciled with a weak energy dependence of the diffusion coefficient, preferred based on measurements of weak CR anisotropy [42-44]. If this were not enough, the spectra of escaping particles are in principle different from the spectra of accelerated particles, and we do not have yet a clear theory of how to connect the two.

The situation does not get any clearer in terms of CR transport in the Galaxy: the simple power law expectation for the spectra of individual chemicals is not confirmed by data by PAMELA [8], and by the more recent AMS-02 data [9], which show a break at rigidity of a few hundred $\mathrm{GV}$ in at least the proton and helium spectra. Such spectra might suggest that the regime of diffusive transport is changing with rigidity, which may for instance happen because of self-generation of waves $[10,11]$ or spatial dependence of the diffusion coefficient [12]. Moreover, the gradient in the $\mathrm{CR}$ pressure may be responsible for launching Galactic winds [13,15], which change in a rather significant way the relation between the injected spectra and the ones observed at the Earth [14] (see [16] for a recent review). None of these effects is usually taken into account in detailed descriptions of CR transport such as GALPROP, while spectral features are typically accommodated by assuming ad hoc breaks in the diffusion coefficient and/or the injection spectra.

Here I will briefly summarize some recent developments and open questions concerning CR acceleration (Sect. 2) and propagation (Sect. 3). I will draw some conclusions in Sect. 4. 


\section{Cosmic Ray acceleration}

While there is a general consensus that SN explosions are somehow related to the origin of Galactic CRs, it is not known as yet which types of SN contribute the most or whether isolated $\mathrm{SNe}$ or superbubbles play a major role, and whether the spectrum at different energies is contributed by one or another class of $\mathrm{SNe}$. The main difference among these different possibilities is not much in the energetics but in the environment in which the explosion takes place, which may be more or less suitable for acceleration to very high energies or for the acceleration of nuclei of different types. In all cases however, at least at the zero order, the spectrum of accelerated particles is expected to be close to $p^{-4}$ (or harder at high energies if non-linear effects are important).

The pivotal point in the physics of CR acceleration is represented by the need for $\mathrm{CR}$ induced magnetic field amplification, namely the enhancement of the scattering frequency of accelerated particles that is self-determined through the excitation of plasma instabilities. This issue is critical in that it may create the conditions for CR acceleration up to very high energies and at the same time explain the morphology of the non-thermal X-ray emission in young SNRs (see [17] for a review). This latter point has represented an observation breakthrough: the detection of spatially thin X-ray filaments of synchrotron emission has allowed us to estimate the magnetic field in the acceleration region, that in most cases turns out to be of order $\sim 100 \mu \mathrm{G}$, thereby supporting the idea of magnetic field amplification and, indirectly, of efficient CR acceleration at the shock.

From the theoretical point of view, the most important progress in the field has been the identification and investigation of the so-called non-resonant-hybrid instability, first described in [4]. The modes excited by this instability have large wavenumber $k_{\max } \gg 1 / r_{L}$, where $r_{L}$ is the Larmor radius of the particles exciting the instability, and very fast growth rate, $\gamma_{\max }=k_{\max } v_{A}\left(v_{A}\right.$ is the Alfvén speed in the background magnetic field). The fast growth of these modes is accompanied by a migration of power in $k$ space towards smaller wavenumbers, until $k \sim 1 / r_{L}^{*}$ (where $r_{L}^{*}=E / e \delta B$ is now the Larmor radius calculated in the amplified magnetic field $\delta B$ ), at which point scattering becomes important and the current of particles producing the instability can be disrupted. In the upstream of a shock, one can envision a self-similar situation as follows: let us consider particles of given energy $E$ returning from downstream. Since there are no waves that can scatter these particles back towards the shock, they escape, thereby creating a current that excites the non-resonant-hybrid instability and exciting the waves necessary for other particles with energy $E$ to scatter back to the shock, where their energy can increase by $\Delta E / E \approx$ $v_{s} / c$. This cycle continues for as long as the instability has enough time to grow. This qualitative picture appears to be nicely confirmed by recent hybrid simulations [18]. This numerical work is leading to unprecedented insights into the physics of DSA.

The application of this instability to type Ia and II supernova explosions was first investigated in [5,6] and later in [7]. The maximum achievable energy as estimated in [7] can be written as

$$
E_{M} \approx 130\left(\frac{\xi_{C R}}{0.1}\right)\left(\frac{M_{e j}}{M_{\odot}}\right)^{-\frac{2}{3}}\left(\frac{E_{S N}}{10^{51} \mathrm{erg}}\right)\left(\frac{n_{I S M}}{\mathrm{~cm}^{-3}}\right)^{\frac{1}{6}} \mathrm{TeV}
$$

for type Ia supernovae, and

$$
\begin{aligned}
E_{M} \approx & 1\left(\frac{\xi_{C R}}{0.1}\right)\left(\frac{M_{e j}}{M_{\odot}}\right)^{-1}\left(\frac{E_{S N}}{10^{51} \mathrm{erg}}\right) \\
& \times\left(\frac{\dot{M}}{10^{-5} M_{\odot} \mathrm{yr}^{-1}}\right)^{\frac{1}{2}}\left(\frac{V_{w}}{10 \mathrm{~km} \mathrm{~s}^{-1}}\right)^{-\frac{1}{2}} \mathrm{PeV}
\end{aligned}
$$

for type II SN explosions in the wind of the progenitor star. Here $\xi_{C R}$ is the CR acceleration efficiency, $M_{e j}$ the mass of the ejecta, $E_{S N}$ the kinetic energy of the explosion. For type Ia $\mathrm{SNe}$, the maximum energy is affected by the density of the upstream medium $n_{I S M}$, while in the second case the relevant density is determined by the mass loss rate $\dot{M}$ and the velocity $V_{w}$ of the wind of the red giant progenitor star. These estimates suggest that $\sim \mathrm{PeV}$ energies can only be reached in the wind scenario and only within the first 20-50 years after the SN explosion, thereby making the direct detection of gamma rays from Galactic PeVatrons quite unlikely.

The scenario depicted above emphasizes the importance of having accelerating particles escaping the shock region from upstream of the shock at the highest energy reached at any given time. The spectrum observed at the Earth after propagation in the Galaxy results from an integration in time of the escape flux during the expansion of the SN shell and the release of particles trapped in the downstream, after the expansion of the $\mathrm{SN}$ shell has come to a stop. Both these contributions are extremely uncertain, and so is the spectrum of CRs injected by an individual SN (see calculations in Refs. $[19,20]$ and a more recent calculation in Ref. [21]).

Multifrequency observations of individual SNRs provide information on the spectrum of CRs advected downstream: in the case of the Tycho SNR, one can make an argument [22] that the local spectrum of accelerated particles, as inferred from gamma ray observations, is somewhat steeper than $E^{-2}$, at odds with both DSA and its non-linear version. Such steeper spectra require either invoking subtle effects associated with the distribution of target material for $p p$ collisions [23] or a substantial role of a finite velocity of scattering centers [22]. Both explanations appear rather unsatisfactory from the theoretical point of view. Independent of the correct explanation of the steep spectrum, gamma ray observations of Tycho require $\mathrm{CR}$ acceleration up to maximum proton energies $\lesssim$ few hundred $\mathrm{TeV}$, compatible with the estimates reported above for $E_{M}$ in a type Ia SN.

Aside from the amplification of the magnetic field upstream of the shock, the non-linearity of DSA manifests itself through the dynamical reaction of accelerated particles on the shock itself: a fluid element approaching the shock from upstream (in the shock frame) encounters an increasing pressure in the form of accelerated particles, thereby being slowed down. This effect leads to the formation of a precursor, in which the velocity of the background plasma gradually decreases while approaching 
the shock discontinuity. The resulting compression factor experienced by accelerated particles becomes a function of their momentum, being smaller at low momenta and larger for high momenta: this is the physical reason for the concave spectra typical of non-linear DSA. Moreover, since part of the total ram pressure $\rho v_{s}^{2}$ is now converted to accelerated particles, there is less energy left for heating the background plasma at the shock, therefore non-linear DSA leads to predicting a lower temperature of the postshock gas.

It is worth recalling that the presence of neutral atomic hydrogen in the acceleration region may potentially lead to modifications in the spectrum of accelerated particles [24] for shock velocities $\lesssim 3000 \mathrm{~km} / \mathrm{s}$. The theory of DSA in the presence of neutrals was recently developed in [24] in the test-particle regime and in [25] in its non-linear version. The spectrum of accelerated particles is modified because of the so-called neutral return flux: neutral hydrogen atoms crossing the (collisionless) shock towards downstream can suffer a charge exchange reaction with a hot ion downstream of the shock. There is a finite probability that the resulting neutral atom moves in the direction of the upstream of the shock, where it can damp its energy and momentum onto the plasma, thereby heating it and slowing it down. This phenomenon leads to a reduction of the Mach number and a to correspondingly steeper spectrum of accelerated particles for momenta such that the diffusion length $D(p) / v_{s} \lesssim \lambda_{n}$, where $\lambda_{n}$ is the pathlength for reactions of charge exchange and ionization. For typical values of the parameters, the effect of neutrals on the spectrum of accelerated particles is limited to $E \lesssim 1-10 \mathrm{TeV}$. The neutral return flux requires that charge exchange reactions downstream occur faster than ionization, a condition that restricts the importance of this phenomenon to shocks moving with velocity $v_{s} \lesssim(3-4) \times 10^{3} \mathrm{~km} / \mathrm{s}$.

The presence of neutrals in the acceleration region also leads to Balmer line emission, which represents a powerful diagnostic tool for testing particle acceleration. In fact the presence of CRs at the shock leads to an enhanced width of the narrow Balmer line (due to charge exchange reactions in the $\mathrm{CR}$ induced precursor discussed above) and to a reduced width of the broad Balmer line (due to the lower gas temperature downstream of the shock that follows from non-linear DSA). These effects are described quantitatively in [25-27], where the appearance of a Balmer line component with intermediate width $\sim$ few hundred $\mathrm{km} / \mathrm{s}$ was also discussed, as resulting from the phenomenon of neutral return flux.

As mentioned above, the Tycho SNR is currently the only case in which we have a rather compelling case for acceleration of CRs up to several hundred TeV energies at a SN shock. Some cases of middle-age SNRs interacting with molecular clouds, such as W44 and IC443 [28], are sometimes indicated as confirmation of SNR shocks as being sources of CRs, because of the detection of the socalled pion bump in the gamma ray spectrum. One should however keep in mind that the multifrequency spectrum of these SNRs is best explained in terms of re-acceleration of ambient, pre-existing CRs, rather than as acceleration from the background gas $[29,30]$. This peculiar nature of such sources is also confirmed by the steep spectra and the relatively low maximum energy of non-thermal particles (in the case of W44 (re)acceleration seems to be turning off at $\sim 100 \mathrm{GeV}$ ).

As discussed above, in special types of SNe (type II in red giant winds) the non-resonant hybrid instability [4] could be excited fast enough to reach the rigidity of the knee. This line of thought leads to the straightforward conclusion that heavier nuclei, with charge $Z e$, may be accelerated to energies $\sim 3 Z \times 10^{15} \mathrm{eV}$ (corresponding to the same rigidity of protons at the knee), namely the spectrum of iron nuclei would extend to $\lesssim 10^{17} \mathrm{eV}$, where Galactic CRs should end (with a predominantly heavy mass composition). This does not exclude that other as yet unknown (and more rare) sources may be contributors to the CR flux up to even higher energies, but at least at present there is no indication that such component does in fact exist. The possibility of an end of the Galactic CR spectrum at energies much lower than the ankle represents a change of paradigm and a source of active debate on the nature of the transition to extragalactic CRs, as well as on the nature of the extragalactic CRs themselves: in fact the combination of data and theory on Galactic CRs and the data on spectrum and mass composition of ultra high energy cosmic rays (UHECRs) as collected by Auger [31], outlines a quite complex and somewhat unappealing picture of the transition region, as recently discussed in [32]: data suggest that the extragalactic CR spectrum is the superposition of different chemical components with hard injection spectrum $\left(\sim E^{-\alpha}\right.$ with $\alpha \sim 1-1.6)$ and maximum rigidity $\lesssim 10^{19} \mathrm{~V}$ and an additional light component (not heavier than helium) with a steep injection spectrum $\left(\sim E^{-2.7}\right)$ also extending up to $\sim(0.5-1) \times 10^{19} \mathrm{eV}$. Such a component has spectrum and normalization compatible with the recently measured spectrum of light elements by KASCADE-Grande [33]. Notice how, with respect to a decade ago, when the main challenge in the physics of UHECRs was to reach maximum (proton) energies $\gtrsim 10^{20} \mathrm{eV}$ in some astrophysical source, nowadays the maximum energy does not appear to be an issue (many classes of sources on the Hillas plot can potentially provide acceleration to $\sim 5 \times 10^{18} \mathrm{eV}$ ), while understanding the mass composition has become the most challenging aspect in this field.

\section{Cosmic Ray propagation}

Where does acceleration end and propagation in the Galaxy begin? Despite our efforts to separate these two phases for the sake of simplicity, the links between them are so strong that it becomes difficult to answer the question above. The phenomenon of particle escape from the sources (see above) well describes this complexity: particles that escape leave the accelerator at a time in which the particle spectrum at the shock is still being forged by the physics of particle acceleration, and in fact it is the escape that regulates the trapping of other particles in the acceleration region. One might argue that at any given time, particles with energy $\gtrsim E$ have left the system and at least for them one could start talking about propagation in the Galaxy. However, as pointed out [34], the self-generation of Alfvén waves by these particles 
affects their propagation near the source, so that such propagation is unlikely to occur with the same properties as the Galactic propagation on much larger scales. I will leave a proper discussion of these effects to a different venue and concentrate below on some issues related to propagation of CRs on Galactic scale.

The most straightforward modelling of CR propagation is based on assuming a spatially constant diffusion coefficient $D(E) \propto E^{\delta}$ throughout the Galactic volume (including the halo of size $H$ ) and an injection rate $Q(E) \propto E^{-\gamma}$ concentrated in a thin Galactic disc of radius $R_{d}$. The spectrum of CRs observed at the Earth, at momenta sufficiently large that energy losses and advection become unimportant $(E>10 \mathrm{GeV})$, can then be estimated as $n(E)=\frac{Q(E)}{2 \pi R_{d}^{2} H} \frac{H^{2}}{D(E)} \sim E^{-\gamma-\delta}$. Hence, if injection is power law-like, as expected from DSA, also the observed spectrum should be a power law, steeper by $\delta$. In principle a scaling like this can be applied to all primary nuclear species.

Measurements of the spectrum of protons and helium nuclei (the most abundant species in CRs) have constantly improved in recent years, and the statistics of events has become large enough to allow for the identification of spectral features: PAMELA has claimed the existence of a hardening in the spectrum of protons and helium nuclei [8], only recently confirmed by AMS-02 [9]. Moreover, both experiments measure a He spectrum that is overall harder than the proton spectrum (in agreement with previous CREAM measurements at higher energies [35]). Finally, a hardening in the spectra of light elements also appears to be necessary to explain the diffuse Galactic gamma ray background [36].

None of these findings is easily accommodated in the standard model of CR propagation, hence the need to look for aspects that may have been overlooked in the basic theory of $\mathrm{CR}$ propagation (although, to be fair, sourcerelated effects cannot be ruled out as yet).

The basic results outlined above are based on pre-assigned, isotropic, homogeneous, one-dimensional diffusion of CRs. Each one of the adjectives used here provides an inaccurate description of Nature. The issue is to understand to which extent.

In the presence of a large scale magnetic field (the Galactic spiral structure provides one), turbulence with an energy-containing scale of $50-100$ pc provides diffusion coefficients in the direction parallel $\left(D_{\|}\right)$and perpendicular $\left(D_{\perp}\right)$ to the ordered field that are very different $\left(D_{\perp}<\right.$ $D_{\|}$even for $\delta B / B \sim 1$ [37]). The asymptotic limit $D_{\|} \approx$ $D_{\perp}=D(E)$ is eventually reached only for $\delta B / B \gg 1$, which does not apply to the Galaxy as a whole, hence the diffusion coefficient $D(E)$ usually adopted in the calculations should be considered as an effective value, to be used to describe some observations. This warning is especially important when dealing with calculations of anisotropies and gradients of the CR distribution. Some attempts are being made at calculating the CR spectrum in the Galaxy retaining the information on anisotropic diffusion (see for instance Ref. [38] for an approach based on stochastic differential equations and Ref. [39] for a discussion of an implementation of anisotropic diffusion in DRAGON).
In addition to being intrinsically anisotropic, diffusion may easily be space dependent: for instance the diffusion coefficient in the halo can have a different energy dependence than the one closer to the Galactic disc. This type of situation has been shown to result in the appearance of spectral breaks [12]: for a given injection rate $Q(E) \propto$ $E^{-\gamma}$, the spectrum observed at the Earth is $\sim E^{-\gamma-\delta_{2}}$ at $E<E_{c}$ and $\sim E^{-\gamma-\delta_{1}}$ at $E>E_{c}$, where $E_{c}$ is a critical energy determined by geometrical factors, and $\delta_{1}$ and $\delta_{2}$ are the slopes of the diffusion coefficient closer and farther away from the disc respectively. If $\delta_{2}>\delta_{1}$, this effect results in a spectral hardening at high energy.

Probably the most problematic aspect of the problem, that is rarely included in calculations of $\mathrm{CR}$ propagation, is the self-generation of waves by the same CRs through streaming instability. This problem was studied in some detail in the ' 70 s $[40,41]$. The old literature on the problem focused on the role of ion-neutral damping that is so effective that Alfvén waves can hardly propagate, thereby leading to quasi-ballistic motion of particles in the neardisc regions [41]. If neutral gas is spatially segregated in dense but small regions (clouds) and most of the volume of the Galaxy is filled with ionized gas, then the growth of waves is balanced by non-linear Landau damping [10]. Within this approach the diffusion coefficient can be calculated self-consistently and typically, at high energy, its energy dependence is related to the slope $\gamma$ of the injection spectrum as $D(E) \sim E^{2 \gamma-3}$. Since the spectrum at the Earth is $n(E) \sim Q(E) / D(E)$, it follows that if the observed spectrum is taken to have a slope 2.7 , the slope at injection is required to be $\gamma \approx 1.9$, not far from the expectation from DSA, especially if non-linear effects due to $\mathrm{CR}$ pressure are taken into account. However, the diffusion coefficient turns out to scale as $D(E) \sim E^{0.8}$, that leads to difficulties in explaining the low levels of anisotropy observed in CRs (see for instance [42-44]), unless rather ad hoc solutions are invoked [52]. The problem is somewhat mitigated if there is pre-existing turbulence that dominates the propagation of CRs at energies above a few $100 \mathrm{GeV}$. When discussing about anisotropy it is however worth keeping in mind that it is a very difficult quantity to calculate and predict, for several reasons: first, the amplitude of the anisotropy observed at the Earth is dominated by the closest and most recent sources (while the CR spectrum is dominated by the large scale distribution of sources), therefore the specific amplitude and phase of the anisotropy and their energy dependence are rather erratic quantities (see for instance [42-44]) since they depend on the realization of sources in which we happen to be located. Second, the local large scale structure of the Galactic magnetic field (namely close to the solar system) may profoundly affect anisotropy and hinder any attempt at pointing at the source location [45]. The difficulty in dealing with anisotropy is confirmed by the unexpected detection of small and medium scale anisotropies by MILAGRO [46] and IceCube [47], that apparently defy the standard theory of diffusive $\mathrm{CR}$ propagation (that only predicts the existence of a dipole) but that could in fact be evidence for local diffusive effects $[48,49]$ or propagation in the heliospheric environment $[50,51]$. 
The combination of pre-existing turbulence in the interstellar medium and self-generated waves gives rise to spectral breaks, and more specifically a hardening at several hundred $\mathrm{GeV}$, that is compatible with the one measured by PAMELA and AMS-02 in the protons and helium spectra $[10,11]$. It is worth stressing that the self-generation scenario leads to the production of Alfvén waves moving only in the direction of the decreasing CR density, so that two conclusions can be drawn: 1) at sufficiently low energy, particle advection with such waves is bound to dominate upon diffusion; typically this happens at energies below $\sim 10 \mathrm{GeV}$ as shown in Ref. [10]; 2) the fact that self-generated waves move only away from the disc implies that there is no reacceleration, therefore this scenario is not compatible with models involving CR reacceleration.

The self-generation is only one of the examples of nonlinear effects induced by CRs that makes the problem of propagation intrinsically complex and fascinating. Another instance (not completely independent on self-generation) is the launching of Galactic winds, as due to the $\nabla P_{C R}$ force at the base of the wind (see pioneering papers in Refs. [13] and [15]). Wherever this force wins over gravity in the direction perpendicular to the Galactic disc, a CR induced wind of ionized gas can be launched, and eventually reach asymptotic velocities of hundreds of $\mathrm{km} / \mathrm{s}$ at several hundred $\mathrm{kpc}$ distance from the disc, passing through a sonic point [13]. The self-generation of waves by CRs (and their damping) plays a crucial role in this problem and is responsible for the formation of the spectrum observed at the Earth [14], as derives from the interaction between self-generated CR diffusion and advection with a CR-launched wind (this topic has recently been reviewed in [16]). In hydrodynamical models of CRinduced winds, CRs are advected away from the base of the wind with a velocity $u_{a} \propto z$, where $z$ is the height above the disc (this scaling is only valid at $z \lesssim$ few tens of $\mathrm{kpc}$ ). Close enough to the disc particles' transport is dominated by diffusion (namely the diffusion coefficient is large) up to some maximum heigh $z_{*}(p)$ (depending on momentum), where advection takes over. At this critical distance, the diffusion time and the advection time are equal:

$$
\frac{z_{*}^{2}}{D(p)} \approx \frac{z_{*}}{u_{a}} \sim \text { constant. }
$$

This implies that $z_{*} \sim D(p)^{1 / 2}$. The critical distance $z_{*}$ plays a role similar to $H$, the size of the halo, in standard diffusion models. Since the standard solution of the diffusion equation scales as $n_{C R}(p) \propto Q(p) H / D(p)$, by analogy one may guess that

$$
n_{C R}(p) \propto Q(p) z_{*} / D(p) \sim Q(p) D(p)^{-1 / 2} \sim E^{-\gamma-\delta / 2},
$$

where $\delta$ is the slope of the diffusion coefficient as a function of momentum. In realistic models, in which diffusion is self-generated, this index is actually determined by the rate of injection of CRs [14] ( $\delta$ is a function of $\gamma$ [16]), but even ignoring this complication it is clear that the presence of a CR induced wind affects profoundly the link between the spectrum of CRs observed at the Earth and the rate of injection, as well as other quantities such as CR anisotropy and CR gradient.

\section{Discussion}

The research on the origin of CRs has been and still is a strongly data-driven field. It is new, better data that allow us to select the best explanation of what Nature is doing. As I wrote in the beginning of this short review, a general picture of the origin of CRs that most of us can agree upon can certainly be found. On the other hand, it is the deviations from this standard lore that are important at the present time, to find hints of missing pieces (there have to be many). Since the general picture of the acceleration and propagation of Galactic CRs is reasonably well outlined, such anomalies are bound to be small, and that makes it difficult to find them observationally. Discrepancies between different experiments measuring the same quantities (often in different ways or with different techniques) are not uncommon in CR physics and should be considered as a warning to have systematic errors and systematic uncertainties under control (to the extent that this is possible at all), especially when new results are being claimed. Spectral features such as the hardenings measured by PAMELA [8] and AMS-02 [9] are a clear instance of such deviations, but it is clear that they must be rather subtle, therefore, despite the impressive statistics of events available, it is the systematic uncertainties that represent the biggest challenge to the realistic assessment of the existence of such features or similar anomalies. As observations become more clear, our theories become better refined and the need to introduce new physical effects rises or fades away.

A similar situation may be presenting itself in the knee region: the need to have efficient particle acceleration of protons up to $E \sim 3 \times 10^{15} \mathrm{eV}$ has been dictated by the detection of a knee in the all-particle CR spectrum. But data on the spectrum of the light component at such energies appear to be problematic: at present it is not clear whether the light CR spectrum (protons plus $\mathrm{He}$ ) has a knee at this energy, as the KASCADE data suggest [53], or rather at somewhat lower energies $(\sim 800 \mathrm{TeV})$, as recently found by ARGO-YBJ [54]. The implications of these different sets of data for the physics of CRs were recently discussed in [7].

At the highest energies the current observational situation is also, in many respects, confusing: one one hand, a flux decrease is apparent at energies $\sim 10^{20} \mathrm{eV}$, which would naturally be expected as a consequence of the onset of photopion production of protons on the photons of the cosmic microwave background. On the other hand, the chemical composition as measured by Auger [31] is not compatible with being proton-dominated in the same energy region. The data of the Telescope Array [55], with all limitations deriving from a poorer statistics of events at the highest energies, seem to be compatible with a light composition. The two sets of data have very different implications on the description of the transition from Galactic to extragalactic CRs as well as on the origin of UHECRs (see [32] for a discussion).

The research of the author is partially funded through Grant PRIN-INAF 2013. 


\section{References}

[1] D. Caprioli, JCAP 5, 26 (2011)

[2] M.A. Malkov and L.O'C. Drury, Rep. Prog. Phys. 64, 429 (2001)

[3] P. Blasi, Astron. \& Astrophys. Rev. 21, 70 (2013)

[4] A.R. Bell, MNRAS 353, 550 (2004)

[5] K.M. Schure \& A.R. Bell, MNRAS 4351174 (2013)

[6] K.M. Schure \& A.R. Bell, MNRAS 4372802 (2014)

[7] M. Cardillo, E. Amato \& P. Blasi, Astropart. Phys. 69, 1 (2015)

[8] O. Adriani et al. (Pamela Coll.), Science 332, 69 (2011)

[9] Data delivered at CERN, http://www.ams02. org/2015/04/ams-days-at-cern-and-latestresults-from-the-ams-experiment-on-theinternational-space-station/

[10] P. Blasi, E. Amato \& P. Serpico, Phys. Rev. Lett. 109, 061101 (2012)

[11] R. Aloisio \& P. Blasi, JCAP 7, 1 (2013)

[12] N. Tomassetti, ApJ Lett. 752, 13 (2012)

[13] D. Breitschwerdt, J.F. McKenzie \& H.J. Völk, A\&A 245, 79 (1991)

[14] V.S. Ptuskin, H.J. Völk, V.N. Zirakashvili \& D. Breitschwerdt, A\&A 321, 434 (1997)

[15] F.M. Ipavich, ApJ 196, 107 (1975)

[16] H.J. Völk, Proceedings of "Cosmic Rays and the InterStellar Medium”, preprint arXiv: 1411.1287

[17] J. Vink, Astron. \& Astrophys. Rev. 20, 49 (2012)

[18] D. Caprioli \& A. Spitkovsky, ApJ Lett. 765, 20 (2013)

[19] V. Ptuskin, V. Zirakashvili \& E-S. Seo, ApJ 718, 31 (2010)

[20] D. Caprioli, E. Amato \& P. Blasi, Astropart. Phys. 33, 160 (2010)

[21] A.R. Bell, MNRAS 447, 2224 (2015)

[22] G. Morlino \& D. Caprioli, A\&A 538, 81 (2012)

[23] E.G. Berezhko, L.T. Ksenofontov \& H.J. Völk, ApJ 763, 14 (2013)

[24] P. Blasi, G. Morlino, R. Bandiera, E. Amato \& D. Caprioli, ApJ 755, 121 (2012)

[25] G. Morlino, P. Blasi, R. Bandiera, E. Amato \& D. Caprioli, ApJ 768, 148 (2013)

[26] G. Morlino, R. Bandiera, P. Blasi \& E. Amato, ApJ 760, 137 (2012)

[27] G. Morlino, P. Blasi, R. Bandiera \& E. Amato, A\&A 558, 25 (2013)

[28] Ackermann et al. (Fermi-LAT Coll.), Science 339, 807 (2013)

[29] Y. Uchiyama, R. Blandford, S. Funk, H. Tajima \& T. Tanaka, ApJ Lett. 723, 122 (2010)
[30] S-H. Lee, D.J. Patnaude, J.C. Raymond, S. Nagataki, P.O. Slane \& D.C. Ellison, Preprint arXiv: 1504.05313

[31] J. Abraham et al. (Pierre Auger Coll.), Phys. Rev. Lett. 104, 091101 (2010)

[32] R. Aloisio, V. Berezinsky \& P. Blasi, JCAP 10, 20 (2014)

[33] W. Apel et al. (KASCADE-Grande Coll.), Phys. Rev. D87, 081101 (2013)

[34] M.A. Malkov, P.H. Diamond, R.Z., Sagdeev, F.A. Aharonian \& I.V. Moskalenko, ApJ 768, 73 (2013)

[35] H.S. Ahn et al. (CREAM Coll.), ApJ Lett. 714, 89 (2010)

[36] D. Gaggero, D. Grasso, A. Marinelli, A. Urbano \& M. Valli, Preprint arXiv: 1504.00227

[37] D. De Marco, P. Blasi \& T. Stanev, JCAP 6, 27 (2007)

[38] F. Effenberger, H. Fichtner, K. Scherer \& I. Büsching, A\&A 547, 120 (2012)

[39] D. Gaggero, L. Maccione, G. Di Bernardo, C. Evoli \& D. Grasso, Preprint arXiv: 1306.6850

[40] J. Skilling, ApJ 170, 265 (1971)

[41] J. Holmes, MNRAS 170, 251 (1975)

[42] A.D. Erlykin \& A.W. Wolfendale, Astropart. Phys. 25, 183 (2006)

[43] V. S. Ptuskin, F. C. Jones, E. S. Seo \& R. Sina, Adv. Space Res. 37, 1909 (1996)

[44] P. Blasi \& E. Amato, JCAP 1, 11 (2012)

[45] P. Mertsch \& S. Funk, Phys. Rev. Lett. 114, 021101 (2015)

[46] A.A. Abdo et al. (MILAGRO Coll.), Phys. Rev. Lett. 101, 221101 (2008)

[47] M. G. Aartsen et al. (IceCube Coll.), Astropart. Phys. 66, 39 (2015)

[48] G. Giacinti \& G. Sigl, Phys. Rev. Lett. 109, 1101 (2012)

[49] M. Ahlers, Phys. Rev. Lett. 112, 1101 (2014)

[50] A. Lazarian \& P. Desiati, ApJ 722, 188 (2010)

[51] L. O'C. Drury, in the proceedings of ICRC2013, Preprint arXiv: 1305.6752

[52] V.N. Zirakashvili, Int. J. Mod. Phys. A 20, 6858 (2005)

[53] W. Apel et al. (KASCADE Coll.), Astropart. Phys. 31, 86 (2009)

[54] I. De Mitri et al. (ARGO-YBJ coll.), in the Proceedings of ISVHECRI 2014, CERN August 2014, Preprint arXiv: 1502.04840

[55] C.C. Jui et al. (Telescope Array Coll.), J. Phys. Conf. Ser. 404, 012037 (2012), Preprint arXiv: 1110.0133 